\title{
EVALUACIÓN DE MATERIALES PARA EL ACOLCHADO DE LA FRESA CULTIVADA BAJO INVERNADERO
}

\section{EVALUATION OF MULCHING MATERIALS ON STRAWBERRY UNDER GREENHOUSE}

\author{
Luz Andrea Calderón Medellín $\bullet$ Diana Carolina Angulo Rivera $\bullet$ Daniel Rodríguez Caicedo² \\ - Carlos Mario Grijalba Rativa' • María Mercedes Pérez Trujillo3,2
}

\section{RESUMEN}

El objetivo fue comparar el efecto de tres materiales de acolchado (cascarilla de arroz, plástico negro y plástico plateado/negro) sobre el crecimiento y rendimiento de las plantas y la calidad de la fruta cosechada en 'Camarosa'. El cultivo fue plantado bajo invernadero de cubierta plástica, empleando un diseño de bloques completos al azar. Mediante muestreos destructivos mensuales se evaluó el área foliar y la materia seca por planta y se ajustó para ambas el modelo de Gompertz. Se realizaron cosechas durante 15 semanas para determinar el rendimiento por planta y la calidad de la fruta en cuatro categorías de clasificación (Extra, I, II y Pérdidas). Se midieron sólidos solubles, calibre y longitud de los frutos. Periódicamente se determinó la temperatura y la humedad gravimétrica del suelo bajo cada tipo de acolchado. Las mayores temperaturas del suelo se registraron en los acolchados plásticos, mientras que la humedad gravimétrica fue similar en todos los materiales. Las plantas acolchadas con plásticos tuvieron similar acumulación de materia seca respecto a las de cascarilla de arroz, sin embargo, el plástico plateado/negro favoreció la mayor área foliar $\left(5647 \mathrm{~cm}^{2} /\right.$ planta). El rendimiento acumulado de las plantas bajo el plástico plateado/negro y la cascarilla de arroz fue similar (347.8 y $279.47 \mathrm{~g} /$ planta), mientras que con el polietileno negro tuvieron el menor rendimiento (246.43 $\mathrm{g} /$ planta), pero con frutos de mayor calibre. El material del acolchado no influyó sobre el contenido de sólidos solubles. Se concluyó que el material más recomendado como acolchado en fresa en las condiciones del estudio fue el plástico plateado/negro.

Palabras clave: polietileno negro, polietileno plateado/negro, cascarilla de arroz, rendimiento, peso seco, área foliar, calidad de la fresa en fresco.

1 Biólogo. Facultad de Ciencias Básicas y Aplicadas, Universidad Militar Nueva Granada, Cajicá

2 Docente Investigador, Facultad de Ciencias Básicas y Aplicadas, Universidad Militar Nueva Granada, Cajicá

3 Autor para correspondencia: maria.perez@unimilitar.edu.co 
Revista FILTAD DE CIENCIAS BẢSICAS 9

\begin{abstract}
This research was aimed at comparing the effect of three mulching materials (rice husks, black polyethylene and silver/black polyethylene) on the plant growth, yield and fruit quality of 'Camarosa'. The experimental crop was planted as a randomized complete block design in a plastic greenhouse. Leaf area and dry matter were measured through monthly destructive samplings and then, these were adjusted to Gompertz model. Harvest was throughout 15 weeks to determine plant yield and fruit quality was carried out by classification in four fruit categories (Extra, I, II and Losses). Soluble solids, size and length of fruits was measured. Soil temperature and gravimetric moisture were frequently measured under mulching treatments. The highest soil temperatures were recorded in synthetic materials, while the gravimetric soil moisture was similar for all treatments. Plants mulched with plastics achieved equal dry matter accumulation than rice husks, however silver/black polyethylene favoured the highest leaf area (5647 cm²/plant). Silver/black plastic and rice husks yield were similar (347.8 y $279.47 \mathrm{~g} /$ plant), while black plastic cover had the lowest yield (246.43 g/plant), but the highest fruit size. Mulching materials did not influence fruit soluble solids. According to this study, silver/black polyethylene cover was the suggested material as mulch in strawberry under greenhouse conditions.
\end{abstract}

Keywords: black polyethylene, silver/black polyethylene, rice husks, yield, dry weight, leaf area, fresh fruit quality.

\title{
INTRODUCCIÓN
}

La producción de fresa en Colombia está destinada principalmente al consumo nacional y a la transformación industrial. Para el año 2011 se registró un área cosechada de fresa de 1135 ha con una producción de 45023 t. Las zonas de mayor producción están ubicadas en los departamentos de Cundinamarca, Antioquia, Norte de Santander, Cauca, Boyacá y Nariño. Actualmente Cundinamarca es el mayor productor de fresa con una participación en el área cosechada nacional del 52.43 \% y una participación en la producción nacional del 63.4\% (Agronet, 2012). El Plan Frutícola Nacional propone dar mayor impulso al cultivo de fresa en Cundinamarca y Antioquia, con incrementos en el área sembrada del 379.3\%, destinando la producción para exportación hacia Estados Unidos, países del Caribe y Europa (Tafur, 2006). Para lograr esto, los investigadores tienen el reto de diseñar sistemas económicos para la producción que mantengan altos rendimientos sin descuidar la calidad de los frutos y que incluyan el uso de recursos reciclables y renovables sin atentar contra el ambiente (Pritts, 2002).

El acolchado es uno de los componentes más importantes en el sistema de producción de la fresa, ya que aporta grandes ventajas para el cultivo respecto al suelo desnudo como la obtención de una cosecha precoz, un mayor rendimiento y la conservación de los niveles de humedad que favorecen el desarrollo de las plantas (Buclon, 1972; Robledo y Vicente, 1988; Tenjo, 2003; Sánchez, 2006). Las cubiertas del suelo minimizan además problemas fitosanitarios puesto que evitan la aparición de malezas y la acumulación de humedad 
en la superficie de las camas manteniendo el fruto limpio y de buena calidad (Sánchez, 2006).

Se han reportado diferencias importantes entre el acolchado orgánico y el de polietileno en cuanto a las propiedades físicas del suelo, crecimiento de las plantas, rendimiento y producción de frutos. Los acolchados orgánicos actúan como fertilizantes de liberación lenta ya que durante su proceso de descomposición incrementan el contenido de materia orgánica en el suelo. La cascarilla de arroz es un material que mantiene una alta humedad en la superficie del suelo y proporciona buena aireación a la zona radicular de las plantas lo que facilita el aprovechamiento de nutrientes y promueve el crecimiento de la planta (Rodríguez, 2007). Por su parte, los acolchados sintéticos mejoran el crecimiento de las plantas y el peso, rendimiento y calidad de los frutos (Singh et al., 2007). La mayoría de películas plásticas tienen la capacidad de aumentar la temperatura del suelo debido a que absorben una proporción de radiación dependiendo las propiedades ópticas del plástico, calentándose y transmitiendo la energía al suelo (Ham et al., 1993; Bonachela et al., 2012). Este incremento en la temperatura del suelo puede ser favorable para el establecimiento de las plantas y la movilización de fotosintatos del tejido vegetativo hacia los frutos (Suárez y Tovar, 1997; Niu et al., 2004; Berglund et al., 2006). Adicionalmente, mejoran la estructura física del suelo facilitando los procesos de intercambio gaseoso, así como la penetración lateral de las raíces (Buclon, 1972).

En Colombia, algunos trabajos relacionados con evaluación de acolchados se han realizado en cultivos de brócoli (Avendaño y Villamizar, 1997), col de bruselas (Suárez y Tovar, 1997), papa (García y Pinilla, 1998) y Gypsophila (Tenjo, 2003). En ellos se ha comparado la temperatura del suelo y el crecimiento, productividad del cultivo y calidad del producto cosechado utilizando principalmente cubiertas plásticas. Sin embargo, la disposición de los desechos plásticos se ha convertido en un serio desafío y por ello se está considerando estudiar otros tipos acolchados más amigables con el ambiente (Laugale et al., 2006). En este trabajo se comparó el efecto de dos tipos de acolchado sintético y uno orgánico sobre el crecimiento, el rendimiento y la calidad de la fruta en fresa (Fragaria $x$ ananassa 'Camarosa') cultivada bajo condiciones de invernadero en Cajicá (Cundinamarca, Colombia), así como sobre la temperatura y humedad del suelo.

\section{MATERIALES Y MÉTODOS}

El estudio se realizó bajo invernadero entre agosto de 2007 y febrero de 2008 en el Laboratorio de Horticultura del Campus Nueva Granada de la Universidad Militar Nueva Granada, ubicado en Cajicá (Cundinamarca, Colombia), a $4^{\circ} 56.705^{\prime}$ N, 7400.704' O y a 2580 m.s.n.m., con plantas del cultivar Camarosa de un año de edad que iniciaban su segundo ciclo de producción. Durante el ensayo se registró una temperatura ambiental promedio de $16.04^{\circ} \mathrm{C}$ y una humedad relativa del $72.76 \%$. En un área de $163.2 \mathrm{~m}^{2}$ se dispusieron nueve camas de $12 \mathrm{~m}$ de largo, $0.6 \mathrm{~m}$ de ancho y $0.4 \mathrm{~m}$ de altura. Las plantas fueron distribuidas al tresbolillo separadas $0.3 \mathrm{~m}$ entre sí. Se utilizó un diseño de bloques completos al azar, con tres tratamientos (plástico negro, plateado/negro y cascarilla de arroz) y tres repeticiones consistentes en parcelas de $10.4 \mathrm{~m}^{2}$ con 61 plantas cada una.

La temperatura y humedad del suelo fueron registradas dos veces por mes. La temperatura del suelo se tomó en tres momentos del día (7:30 a.m., 11:00 a.m. y 2:30 p.m.), empleando un termómetro de punzón insertado en un orificio alrededor de la planta a $15 \mathrm{~cm}$ de profundidad. La humedad del suelo se determinó por el método 
gravimétrico descrito por Montenegro y Malagón (1990).

El crecimiento de las plantas se evaluó mediante muestreos destructivos mensuales de tres plantas por tratamiento, a razón de una por parcela. Se tomó el peso seco total por planta, secando las muestras en horno a $70^{\circ} \mathrm{C}$ durante 72 horas. Se midió el área foliar por planta empleando el método no destructivo propuesto por Naranjo et al., (2007), el cual consistió en medir el ancho de cada uno de los foliolos, asumiendo que equivale al diámetro de un círculo, calcular el área de dicho círculo y transformar este valor en área foliar mediante la ecuación (1):

\section{$Y=0.8823 X+4.0761$}

Donde $Y$, es el área foliar de cada foliolo y $X$ corresponde al área del círculo, ambas medidas en $\mathrm{cm}^{2}$.

Cuando las plantas iniciaron la etapa de producción, los frutos en estado de madurez 6, según la Carta de Color de la NTC 4103 (ICONTEC, 1996), fueron cosechados semanalmente y se obtuvo su peso fresco por parcela. Todos los frutos se clasificaron en las categorías de calidad establecidas para la fresa (Extra, I y II, según ICONTEC, 1996), y se consideró una adicional de Pérdidas, que correspondió a fruta de tamaño muy pequeño o que estaba afectada por problemas fitosanitarios. La medición de los sólidos solubles se realizó a los 145, 173 y 180 días después de la poda (DDP), tomando una muestra de tres frutos en punto de cosecha por parcela y para cada categoría de calidad, los cuales fueron macerados y cuyo jugo fue analizado empleando un refractómetro digital. El calibre y la longitud de la fruta cosechada fueron medidos para cada tratamiento y parcela, a partir de una muestra de tres frutos de cada categoría de calidad, empleando un calibrador.

Los resultados obtenidos se sometieron a un análisis de varianza utilizando el programa SAS versión 9.00. Se evaluó el supuesto de normalidad de residuales por medio de la prueba de Shapiro-Wilk la cual fue aceptada en todas las variables evaluadas excepto para la temperatura del suelo a las 7 a.m. y el calibre de los frutos, valores que se corrigieron al transformar los datos por la función logaritmo. Se utilizó la prueba de Tukey para las comparaciones múltiples.

Mediante el software R versión 2.6.2 se obtuvieron las curvas de acumulación de peso seco total y de área foliar por planta. Se ajustó el modelo de crecimiento de Gompertz mediante la ecuación (2):

$$
y=\alpha e^{-e^{-k(t-\phi)}(2)}
$$

Donde $\alpha$, es la asíntota superior o el máximo crecimiento, cuyo estimado es $a ; \phi$ es el tiempo en el que se logra la máxima tasa de crecimiento, estimado como g; y k es un parámetro de escala sobre el tiempo $t$ que influye en la tasa de crecimiento (Seber y Wild, 2003). El ajuste del modelo se determinó mediante el cálculo del $R^{2}$ ajustado, según lo propuesto en IDREUCLA (2013) para modelos no lineales. Se efectuó la comparación de los parámetros a, k y $g$ de este modelo obtenidos para los diferentes tratamientos de acolchado, mediante análisis de varianza y pruebas de Tukey, empleando el software R versión 2.6.2.

\section{RESULTADOS Y DISCUSIÓN}

\section{Temperatura del suelo}

Se encontró un efecto diferencial de los materiales del acolchado sobre la temperatura del suelo para los tres momentos del día: 7:30 a.m. $(p<0.0001), 11: 00$ a.m. $(p=0.0004)$ y 3:00 p.m. $(p<0.0001)$ (Tabla 1). 
Tabla 1. Temperatura promedio del suelo durante las horas de muestreo $\left({ }^{\circ} \mathrm{C}\right)$.

\begin{tabular}{|c|c|c|c|}
\hline \multirow[t]{2}{*}{ Tratamiento } & \multicolumn{3}{|c|}{ Hora del día } \\
\hline & $\begin{array}{l}\text { 7:30 } \\
\text { a.m. }\end{array}$ & $\begin{array}{c}11: 00 \\
\text { a.m. }\end{array}$ & $\begin{array}{l}\text { 3:00 } \\
\text { p.m. }\end{array}$ \\
\hline Plástico negro & $17.02 \mathrm{a}$ & $17.85 a$ & $18.37 \mathrm{a}$ \\
\hline $\begin{array}{l}\text { Plástico } \\
\text { plateado }\end{array}$ & $16.72 \mathrm{a}$ & $17.55 \mathrm{a}$ & $18 a$ \\
\hline $\begin{array}{c}\text { Cascarilla } \\
\text { de arroz }\end{array}$ & $16.17 b$ & $16.83 \mathrm{~b}$ & $16.98 b$ \\
\hline
\end{tabular}

Diferentes letras en una misma columna indican diferencias significativas entre tratamientos $p<0.05$

Los resultados mostraron un incremento importante en la temperatura del suelo de las camas de fresa acolchadas con las láminas de polietileno frente a las de cascarilla de arroz. Cabe anotar que las temperaturas más altas en el suelo se registraron a las 3:00 p.m. en los tres tipos de acolchado.

El incremento en la temperatura del suelo obtenido con los acolchados plásticos concuerda con las características de estos materiales, que al ser permeables a las radiaciones infrarrojas o caloríficas (Robledo y Vicente, 1988), presentan durante el día una absorción del $50 \%$ de la radiación, reteniendo el calor del sol y transmitiéndolo al suelo, por lo que se incrementa la temperatura bajo el plástico. Durante la noche el plástico sigue irradiando a la vez que evita que este calor y el producido por las plantas sea liberado al ambiente (Ibarra y Rodríguez, 1991; Tenjo, 2003; Kwabiah, 2004).

El resultado obtenido en esta investigación coincide con lo reportado por De Souza e Igue (1973) y Ramakrishna et al., (2006), en cultivos de cacahuetes y fresa 'Campinas' respectivamente, quienes encontraron que el polietileno logra mayores incrementos en la temperatura del suelo frente a la cascarilla de arroz. Contrario a lo que se esperaba, el color de las láminas plásticas no marcó una diferencia estadística en la temperatura del suelo para ninguna de las horas evaluadas durante el ensayo. Según Suárez y Tovar (1997), la coloración del acolchado tiene un efecto sobre la energía o albedo; la temperatura del suelo se ve afectada por este aspecto ya que colores oscuros absorben una mayor cantidad de radiación que láminas de coloraciones claras y tienden a calentarse en mayor medida.

\section{Humedad gravimétrica del suelo}

La humedad gravimétrica del suelo se comportó de forma similar en los tres tratamientos ( $p=0.9742)$ (Tabla 2). A pesar de no realizar comparaciones de la humedad gravimétrica del suelo desnudo frente a la del suelo cubierto, se encontró que los tres materiales fueron capaces de conservar los niveles de humedad en igual medida, siendo alrededor del 25\% para todos los acolchados. Este valor se encuentra dentro del rango de humedad aprovechable para las plantas que fue determinado específicamente para el tipo de suelo donde se realizó el experimento (González et al., 2009).

Tabla 2. Porcentaje de humedad gravimétrica promedio del suelo de acuerdo al material de acolchado.

\begin{tabular}{cc}
\hline Tratamiento & Humedad (\%) \\
\hline Cascarilla de arroz & $24.99 \mathrm{a}$ \\
\hline Plástico plateado/negro & $25.18 \mathrm{a}$ \\
\hline Plástico negro & $25.03 \mathrm{a}$ \\
\hline
\end{tabular}

Diferentes letras en las columnas indican diferencias significativas entre tratamientos $p<0.05$

\section{Área foliar y materia seca}

El área foliar de las plantas de fresa cultivadas sobre la cascarilla de arroz, el plástico planteado/negro y el plástico negro, se presenta en las Figuras 1A, 1B y $1 \mathrm{C}$, respectivamente, donde se muestran tanto los datos observados como la curva ajustada con el modelo de Gompertz para cada tratamiento de acolchado. 
Las plantas de fresa que fueron acolchadas con el plástico plateado/negro, presentaron la máxima área foliar (a), siendo superior a la encontrada en las plantas sobre cascarilla de arroz y plástico negro (Tabla 3).

Tabla 3. Valores de los parámetros del modelo de Gompertz para el área foliar en plantas de fresa (Fragaria $x$ ananassa 'Camarosa') para cada tipo de acolchado.

\begin{tabular}{cccc}
\hline Tratamiento & $\mathbf{a}$ & $\mathbf{k}$ & $\mathbf{g}$ \\
\hline Cascarilla de arroz & $4849 \mathrm{~b}$ & $0.042 \mathrm{a}$ & $40.66 \mathrm{a}$ \\
\hline $\begin{array}{c}\text { Plástico } \\
\text { Plateado/negro }\end{array}$ & $5647 \mathrm{a}$ & $0.033 \mathrm{a}$ & $37.30 \mathrm{a}$ \\
\hline Plástico Negro & $4747 \mathrm{~b}$ & 0.036 a & 44.26 a \\
\hline
\end{tabular}

- $A F=a \cdot \exp ^{(-\exp (-k \cdot(D D S-g)))} \cdot a:$ máxima área foliar; $k$ : parámetro de escala sobre el tiempo DDT que influencia la tasa de crecimiento; g: tiempo en el que se logra la máxima tasa de crecimiento (Seber and Wild, 2003). AF: Área foliar ( $\mathrm{cm}^{2} /$ planta); DDP: Días después de poda. Diferentes letras en una misma columna indican diferencias significativas entre tratamientos $p<0.05$.

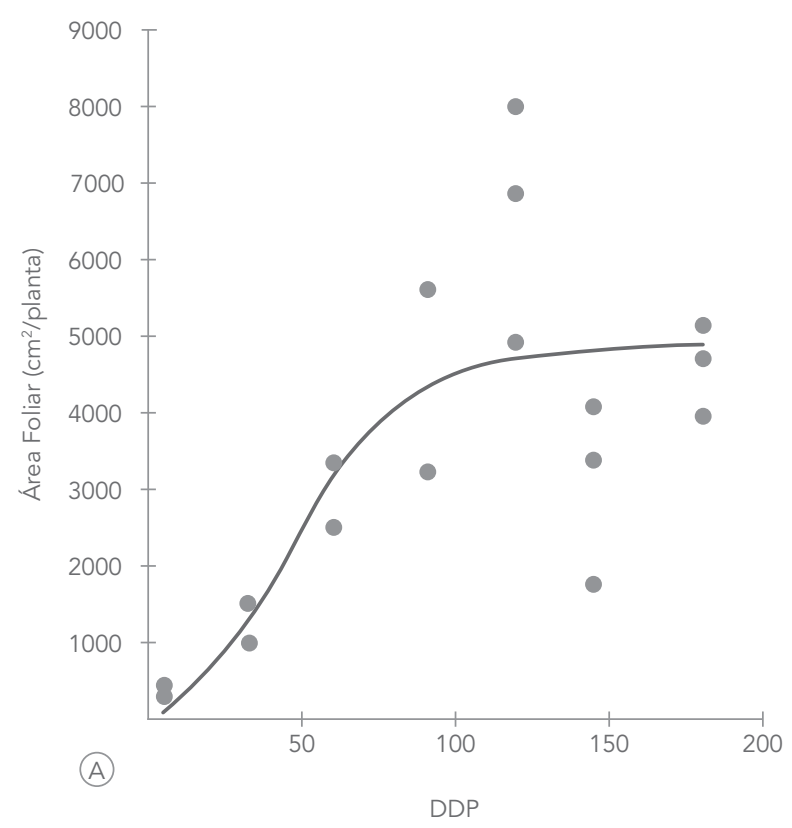

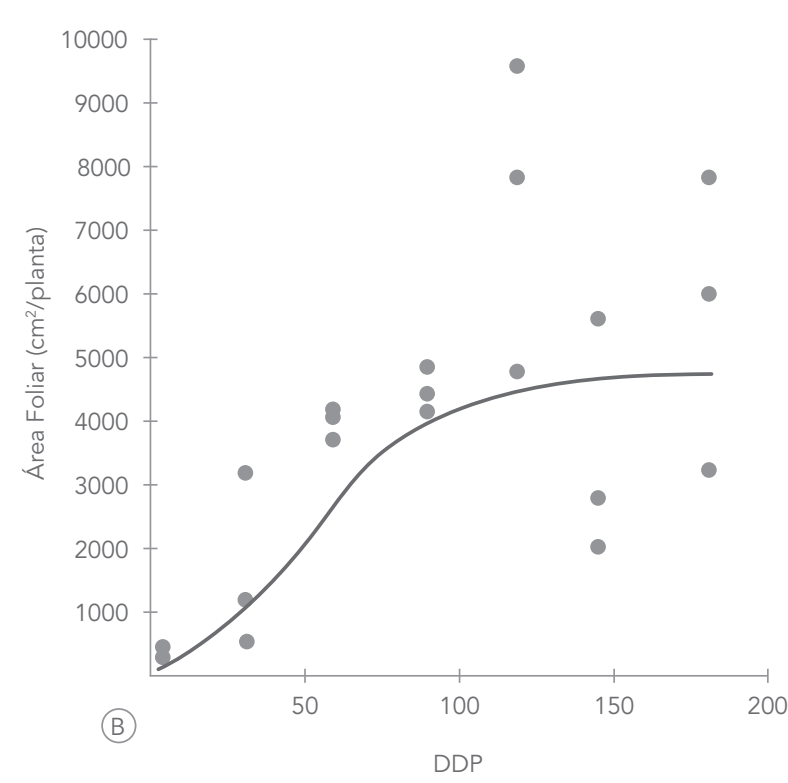

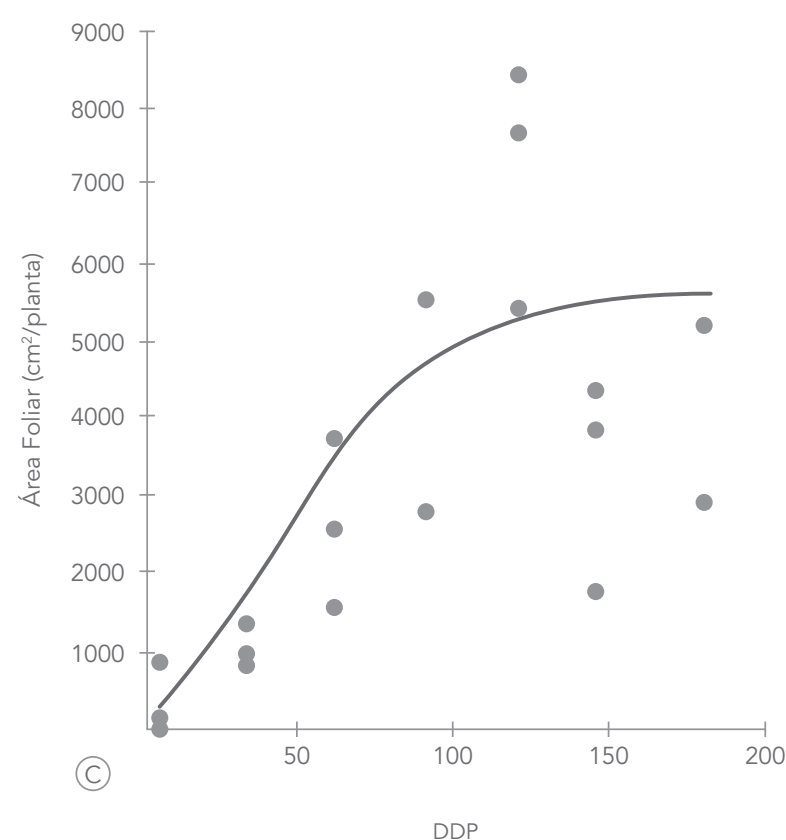

Figura 1. Área foliar acumulada por planta de fresa (Fragaria $x$ ananassa 'Camarosa') para cada tipo de acolchado. Datos observados y modelo de Gompertz. A. Cascarilla de arroz; AF $\sim 4849 \cdot \exp ^{(-\exp (-0.042 \cdot(\mathrm{DDP}-40.66)) ;} ; R^{2}$ ajustado: 0.64. B. Plástico negro; $A F \sim 4747 \cdot \exp ^{(-\exp (-0.036 \cdot(\mathrm{DDP}-44.26)) ;} \mathrm{R}^{2}$ ajustado: 0.53. C. Plástico plateado/negro; AF $5647 \cdot \exp \left(-\exp (-0.033 \cdot(\mathrm{DDP}-37.3)) ; \mathrm{R}^{2}\right.$ ajustado: 0.56. AF: Area foliar ( $\mathrm{cm}^{2} / \mathrm{planta)}$; DDP: Días después de la poda. 
En cuanto a la materia seca acumulada por planta obtenida en los diferentes tratamientos de acolchado, los datos observados y las curvas ajustadas siguiendo el modelo de Gompertz, se presentan en las Figuras 2A, 2B y 2C, para la cascarilla de arroz, el plástico plateado/negro y el plástico negro, respectivamente. Ante los tratamientos de acolchado evaluados, las plantas de fresa no presentaron diferencias en la máxima materia seca acumulada (a), ni en el tiempo en que presentaron la máxima tasa de crecimiento (g) (Tabla 4).

Tabla 4. Valores de los parámetros del modelo de Gompertz para el peso seco total por planta en fresa (Fragaria $x$ ananassa 'Camarosa') para cada tipo de acolchado.

\begin{tabular}{cccc}
\hline Tratamiento & $\mathbf{a}$ & $\mathbf{k}$ & $\mathbf{g}$ \\
\hline $\begin{array}{c}\text { Cascarilla } \\
\text { de arroz }\end{array}$ & $155.4 \mathrm{a}$ & $0.025 \mathrm{a}$ & $3.94 \mathrm{a}$ \\
\hline $\begin{array}{c}\text { Plástico } \\
\text { Plateado/negro }\end{array}$ & $225.6 \mathrm{a}$ & $0.016 \mathrm{a}$ & $28.32 \mathrm{a}$ \\
\hline Plástico Negro & $224.8 \mathrm{a}$ & $0.015 \mathrm{a}$ & $37.99 \mathrm{a}$ \\
\hline
\end{tabular}

Peso Seco $\sim a \cdot \exp (-\exp (-k \cdot(D D T-9)))$ a: máximo peso seco total; $k$ : parámetro de escala sobre el tiempo DDT que influencia la tasa de crecimiento; g: tiempo en el que se logra la máxima tasa de crecimiento (Seber and Wild, 2003). PS: Peso seco (g/planta); DDP: días después de la poda. Diferentes letras en una misma columna indican diferencias significativas entre tratamientos $\mathrm{p}<0.05$.

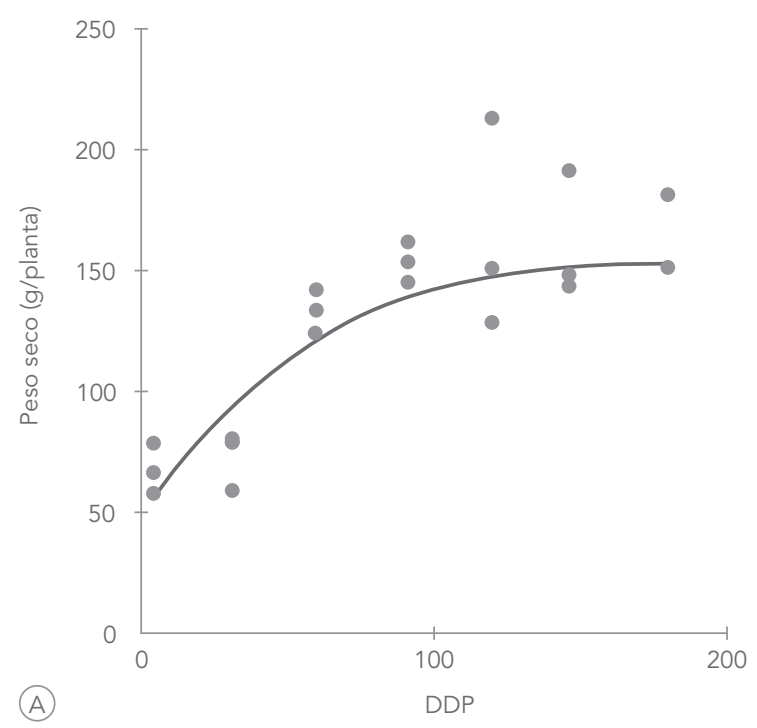

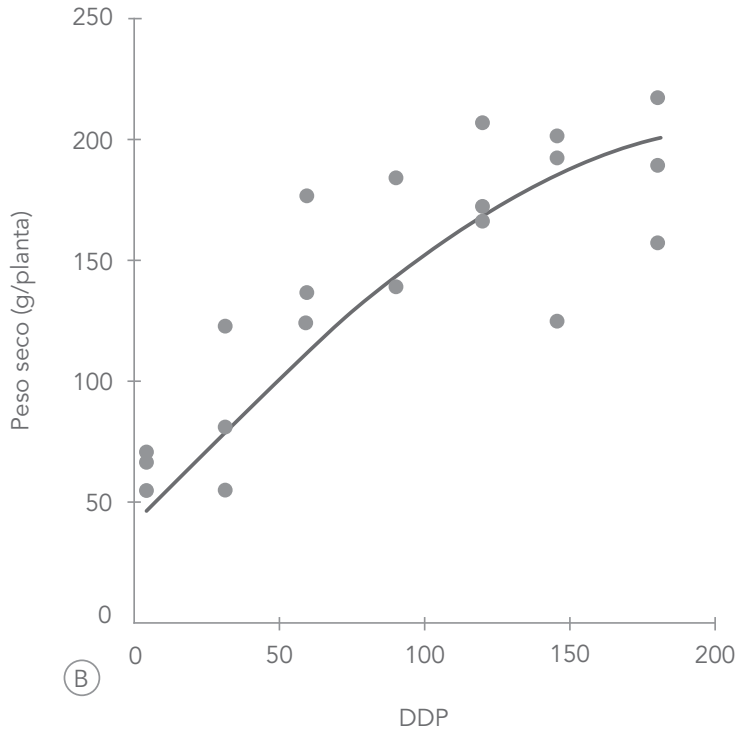

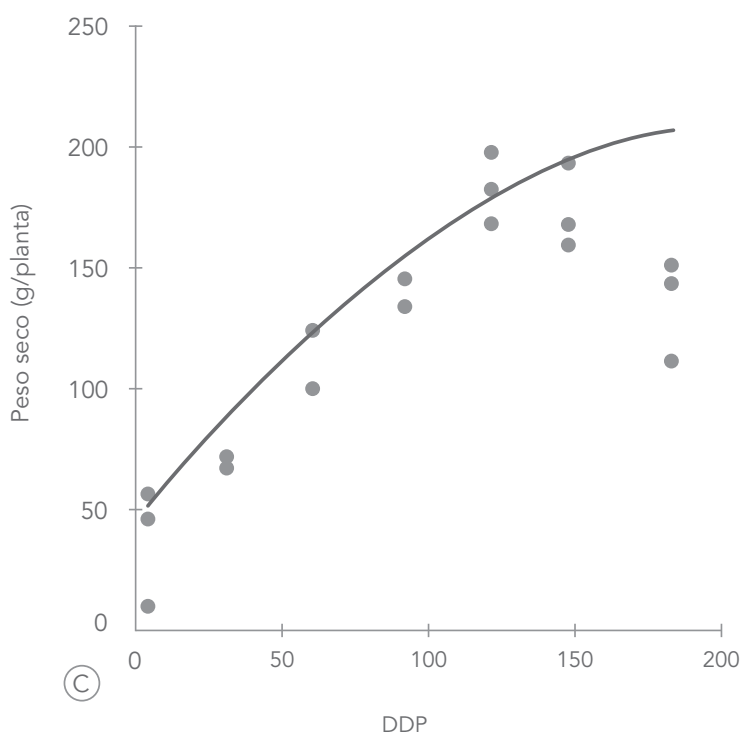

Figura 2. Curvas de acumulación de materia seca por planta de fresa (Fragaria $x$ ananassa 'Camarosa') para cada tipo de acolchado. Datos observados y modelo de Gompertz. A. Cascarilla de arroz; PS 155.4

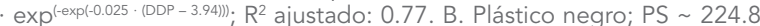

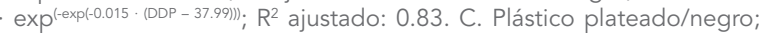
PS 225.6 $\cdot \exp \left(-\exp (-0.016 \cdot(D D P-20.32)) ; R^{2}\right.$ ajustado: 0.76. PS: Peso seco (g/ planta); DDP: Días después de la poda. 
El efecto favorable que tuvo el acolchado plateado/negro sobre el área foliar de las plantas de fresa, se puede explicar por la reflexión total del espectro visible y parcial del UV por el efecto de los materiales plásticos con superficies claras, la cual está relacionada directamente con una mayor radiación fotosintéticamente activa, permitiendo en las plantas un mejor aprovechamiento de la luz y estimulando su crecimiento y desarrollo (Brazanti, 1989; Tenjo, 2003; Poling, 2005). Los plásticos metalizados de superficie rugosa dispersan la luz en diferentes direcciones, creando un entorno más luminoso alrededor de la planta, lo que genera mayor precocidad y rendimiento (Robledo y Vicente, 1988; Avendaño y Villamizar, 1997; Poling, 2005).

En las curvas obtenidas para el peso seco, se observó una tendencia de mayor acumulación en las plantas sobre los acolchados plásticos en comparación a la cascarilla de arroz. Esto puede estar relacionado con la mayor acumulación del $\mathrm{CO}_{2}$ que se forma a partir de la respiración de los órganos subterráneos de la planta y de la actividad biológica en el suelo, haciendo que este se mantenga debajo del plástico y se acumule en niveles altos. El gas es expulsado al ambiente a través de los agujeros del plástico donde se encuentran las plantas, creándose un "efecto chimenea" que favorece el desarrollo de las hojas y de los demás órganos de la planta (Robledo y Vicente, 1988; Tenjo, 2003).

\section{Rendimiento}

El tipo de material empleado para el acolchado de la fresa 'Camarosa' influyó significativamente en la producción de frutos $(p=0.0271)$, encontrándose que las plantas más productivas crecieron sobre el plástico plateado/negro cosechando 24.45 y $41.13 \%$ más peso de frutos que aquellas acolchadas con cascarilla de arroz y plástico negro, respectivamente (Tabla 5).

El comportamiento de la fresa bajo el plástico plateado/negro coincide con Suárez y Tovar (1997) para
Tabla 5. Rendimiento neto acumulado por planta de fresa (Fragaria $x$ ananassa 'Camarosa') para cada tipo de acolchado en 15 semanas de cosecha.

\begin{tabular}{cc}
\hline Tratamiento & $\begin{array}{c}\text { Peso fresco fruta } \\
\text { cosechada (g/planta) }\end{array}$ \\
\hline Plástico plateado/negro & $347.80 \mathrm{a}$ \\
\hline Cascarilla de arroz & $279.47 \mathrm{ab}$ \\
\hline Plástico negro & $246.43 \mathrm{~b}$ \\
\hline
\end{tabular}

Diferentes letras en la columna indican diferencias significativas entre tratamientos $p<0.05$.

col de bruselas y por Poling (2005) para fresa, quienes reportan una mayor productividad en las plantas acolchadas con este tratamiento frente al plástico negro. Con el plástico negro se obtuvo la menor productividad, incluso menor a la alcanzada con la cascarilla de arroz, siendo acorde a lo encontrado por De Souza e Igue (1973). Lo anterior posiblemente se debe al color de este material, el cual absorbe una gran cantidad de luz pero refleja menos del $50 \%$, por lo que la radiación generada alrededor de la planta es menor respecto a la reflejada por las superficies más claras como el polietileno plateado/negro e incluso por la cascarilla de arroz y como resultado se obtiene una productividad más baja (Robledo y Vicente, 1988).

Por otra parte, Branzanti (1989), afirma que el número de coronas tiene un efecto determinante en la productividad de un cultivo de fresa, dado que las plantas están en la capacidad de formar y sustituir coronas secundarias a lo largo de su ciclo vegetativo y cada una de estas origina tallos, estolones y racimos florales. De esta forma, también se explica por qué las plantas acolchadas con plástico plateado/negro alcanzaron una mayor productividad respecto a los demás tratamientos, ya que este material fue el que favoreció el mayor número de coronas por planta (13.11), seguido de la cascarilla de arroz (11.67) y del plástico negro (10). 
A pesar que las plantas de fresa bajo los diferentes materiales de acolchado presentaron valores similares en cuanto a su peso seco, el área foliar fue mayor en aquellas con el plástico plateado/negro. Lo anterior puede indicar que en este tratamiento las plantas tuvieron una mayor interceptación de luz para el proceso de fotosíntesis, lo que contribuiría a explicar su mayor rendimiento (Taiz y Zeiger, 2006).

En cuanto a la influencia del material de acolchado sobre la calidad comercial de la fruta cosechada, se encontraron diferencias estadísticas respecto a la cantidad de ésta que fue clasificada en las diferentes categorías de calidad: Extra, I y ||, con $p=0.0043$, $p=0.0549$ y $p=0.0041$, respectivamente. Las plantas de fresa acolchadas con el plástico plateado/negro produjeron más cantidad de fruta en las categorías Extra y II respecto a los demás tratamientos. Para las Pérdidas se produjeron cantidades similares de frutos en los tres tratamientos evaluados ( $p=0.1463$ ) (Tabla 6).

Por otra parte, los acolchados no influyeron en el contenido de sólidos solubles para los frutos de las categorías Extra $(p=0.4838), \quad$ I $(p=0.8756)$ ni $\|$ $(p=0.1779)$ (Tabla 7).

En promedio el contenido de azúcar para los frutos de fresa 'Camarosa' fue de $7.33^{\circ}$ Brix, encontrándose

Tabla 6. Peso fresco acumulado de frutos de fresa (g/planta) (Fragaria $x$ ananassa 'Camarosa') clasificada en las diferentes categorías de calidad para cada tipo de acolchado durante 15 semanas de cosecha.

\begin{tabular}{ccccc}
\hline Tratamiento & \multicolumn{4}{c}{ Categoría de calidad } \\
\hline & Extra & I & II & Pérdidas \\
\hline $\begin{array}{c}\text { Plástico } \\
\text { plateado/negro }\end{array}$ & $\begin{array}{c}118.53 \\
\text { a }\end{array}$ & $\begin{array}{c}114.25 \\
\text { a }\end{array}$ & $\begin{array}{c}115.02 \\
\text { a }\end{array}$ & $\begin{array}{c}110.54 \\
\text { a }\end{array}$ \\
\hline $\begin{array}{c}\text { Cascarilla } \\
\text { de arroz }\end{array}$ & $\begin{array}{c}98.59 \\
\text { b }\end{array}$ & $\begin{array}{c}90.15 \\
\text { ab }\end{array}$ & 90.73 b & 103.91 a \\
\hline Plástico negro & 81.33 b & 78.04 b & 87.07 b & 80.12 a \\
\hline
\end{tabular}

Diferentes letras en una misma columna indican diferencias significativas entre tratamientos $p<0.05$.
Tabla 7. Contenido de sólidos solubles de los frutos de fresa (Fragaria $x$ ananassa 'Camarosa') para cada tipo de acolchado.

\begin{tabular}{cccc}
\hline Tratamiento & \multicolumn{3}{c}{$\begin{array}{c}\text { Sólidos Solubles } \\
\text { ('Brix) }\end{array}$} \\
\hline & Extra & I & II \\
\hline Cascarilla de arroz & 7,12 a & 8,31 a & 7,63 a \\
\hline Plástico negro & 6,64 a & 7,27 a & 7,62 a \\
\hline Plástico plateado/negro & 7,14 a & 7,48 a & 6,75 a \\
\hline
\end{tabular}

Diferentes letras en una misma columna indican diferencias significativas entre tratamientos $p<0,05$.

por encima del valor mínimo (7.0) de Sólidos Solubles Totales exigido por la industria para la comercialización de la fresa (Camacho, 1988 citado en Gallo y Santacruz, 1996). Bautista (1977), afirma que los sólidos solubles en los frutos están influenciados por factores externos cuyo efecto se da durante las primeras fases del crecimiento, mientras que la fase previa a la maduración está influenciada por factores internos, tales como mecanismos enzimáticos de desdoblamiento y síntesis de compuestos orgánicos. Según Cajuste et al., (2002) y Bautista (1977), una alta luminosidad, altas temperaturas diurnas, bajas temperaturas nocturnas y una baja precipitación favorecen el incremento de azúcares en los frutos.

El tipo de acolchado influyó significativamente en la longitud de los frutos de fresa de las calidades Extra $(p=0.0055)$ y I $(p=0.0224)$. Las plantas acolchadas con plástico negro presentaron frutos de calidad Extra de mayor longitud, mientras que para la calidad I los frutos más pequeños pertenecieron al plástico plateado/ negro (Tabla 8).

Los frutos de la categoría Extra fueron los únicos en los que se presentaron diferencias significativas en el calibre de acuerdo al tipo de acolchado ( $p=0.0027)$, resultando favorecido por los tratamientos plásticos (Tabla 9).

El tamaño de los frutos que fueron cosechados de las plantas acolchadas con el plástico negro resultó ligeramente superior al obtenido con los otros materiales. 
Tabla 8. Longitud promedio de frutos de fresa (Fragaria $x$ ananassa 'Camarosa') según su calidad (Extra, I y II), para cada tipo de acolchado.

\begin{tabular}{cccc}
\hline Tratamiento & \multicolumn{2}{c}{ Longitud del fruto(cm) } \\
\hline & Extra & I & II \\
\hline Cascarilla de arroz & 4.58 a & 3.90 a & 3.26 a \\
\hline Plástico negro & $4.78 \mathrm{~b}$ & $3.88 \mathrm{a}$ & $3.24 \mathrm{a}$ \\
\hline $\begin{array}{c}\text { Plástico } \\
\text { plateado/negro }\end{array}$ & $4.56 \mathrm{a}$ & $3.78 \mathrm{~b}$ & $3.17 \mathrm{a}$ \\
\hline
\end{tabular}

Diferentes letras en una misma columna indican diferencias significativas entre tratamientos $p<0.05$.

Tabla 9. Calibre promedio de los frutos de fresa (Fragaria $x$ ananassa 'Camarosa') según su calidad (Extra I y II), para cada tipo de acolchado.

\begin{tabular}{cccc}
\hline Tratamiento & \multicolumn{3}{c}{ Calibre (cm) } \\
\hline & Extra & I & II \\
\hline Cascarilla de arroz & $3.29 \mathrm{a}$ & $2.79 \mathrm{a}$ & $2.32 \mathrm{a}$ \\
\hline Plástico negro & $3.47 \mathrm{~b}$ & $2.87 \mathrm{a}$ & $2.35 \mathrm{a}$ \\
\hline $\begin{array}{c}\text { Plástico } \\
\text { plateado/negro }\end{array}$ & $3.40 \mathrm{~b}$ & $2.85 \mathrm{a}$ & $2.26 \mathrm{a}$ \\
\hline
\end{tabular}

Diferentes letras en una misma columna indican diferencias significativas entre tratamientos $p<0.05$.

Este último resultado podría atribuirse a la competencia entre los vertederos de las plantas, ya que bajo este tratamiento produjeron una menor cantidad de frutos pero de mayor tamaño en comparación a aquellos en los otros tratamientos (Taiz y Zeiger, 2006). Lo anterior coincide con Singh et al., (2007) quien encontró que el tamaño de los frutos de fresa 'Chandler' del tratamiento con polietileno negro fue superior al de los tratamientos con plástico transparente y cascarilla de arroz.

Los anteriores resultados contrastan ampliamente con lo encontrado por Casierra et al., (2011), quienes en condiciones del trópico alto compararon diferentes colores de plásticos para el acolchado de la fresa 'Ventana', demostrando la superioridad en crecimiento y rendimiento de las plantas cultivadas sobre las películas negras y rojas frente a las plateadas. Estos autores obtuvieron muy baja área foliar y consecuentemente menor rendimiento y tamaño de fruta, en plantas que se desarrollaron sobre películas aluminizadas. Lo anterior lo atribuyen a la alta cantidad de radiación UV que es reflejada por el polietileno plateado, causando restricciones a la expansión foliar y quemazones en los márgenes de las hojas, situaciones que no se presentaron en esta investigación.

\section{CONCLUSIONES}

El plástico plateado/negro fue el material más apropiado para utilizar como acolchado en el cultivo de fresa 'Camarosa' en las condiciones del experimento bajo invernadero, ya que favoreció una mayor área foliar, mayor número de coronas y mayor rendimiento.

Los materiales plásticos favorecieron el incremento de la temperatura del suelo en mayor medida que la cascarilla de arroz.

Los acolchados evaluados ejercieron un efecto similar sobre la humedad gravimétrica del suelo.

El uso de cascarilla de arroz como material para acolchar cultivos es aconsejable si se busca establecer un cultivo orgánico y amigable con el ambiente, lo cual se evidenció en este trabajo teniendo en cuenta que las plantas acolchadas con este material alcanzaron un rendimiento estadísticamente similar al logrado con el plástico plateado/negro. Además, luego de finalizar el ciclo de cultivo, este material se puede incorporar en el terreno como fuente de materia orgánica.

\section{AGRADECIMIENTOS}

A la Facultad de Ciencias Básicas y Aplicadas de la Universidad Militar Nueva Granada por la financiación de este proyecto, a los auxiliares de campo, Sr. Alex Díaz y Sr. Nelson Díaz y al Doctor Edgar Benítez por el apoyo en el análisis estadístico. 


\section{BIBLIOGRAFÍA}

1 Avendaño S y Villamizar G. 1997. Evaluación del efecto de acolchados plásticos y gallinaza en el crecimiento y rendimiento del cultivo de brócoli (Brassica oleracea L. variedad Halica) en la Sabana de Bogota. Tesis, Programa de Ingeniería Agronómica, Facultad de Agronomía, Universidad Nacional de Colombia, Bogotá, D. C.

2 Agronet. 2012. Área cosechada, producción y rendimiento de fresa, 1992-2011. Ministerio de Agricultura y Desarrollo Rural. http://www.agronet.gov. co/agronetweb1/Estad\%C3\%ADsticas.aspx, consulta Octubre de 2013.

3 Bautista D. 1977. Influencia de la temperatura, la insolación y la precipitación sobre los sólidos solubles del fruto de la mora (Rubus glaucus Benth). Agronomía Tropical, 28(4): 399-407.

4 Berglund R, Svensson B y Gertsson U. 2006. Impact of plastic mulch and poultry manure on plant establishment in organic strawberry production. Journal of plant nutrition, 29: 103-112.

5 Bonachela S, Granados MR, López JC, Hernández J, Magán JJ, Baeza EJ y Baille A. 2012. How plastic mulches affect the thermal and radiative microclimate in an unheated low-cost greenhouse. Agricultural and Forest Meteorology, 152: 65-72.

6 Branzanti EC. 1989. Capítulo 3: La 'planta' fresa. p. 49-58 En: La fresa. Editorial Mundi- Prensa, España. 386 p.

7 Buclon F. 1972. Le paillage plastique: principes d'utilisation en culture du fraiser. Symposium on Strawberry under Protection. Acta Horticulturae, 30: 53-59.

8 Cajuste J, López L, Rodríguez J y Reyes M. 2002. Caracterización fisicoquímica de tres cultivares introducidos de zarzamora erecta (Rubus sp.). Fundación Salvador Sánchez Colin, CICTAMEX, México, 6: 26-33.
9 Casierra-Posada F, Fonseca E y Vaughan G. 2011. Fruit quality in strawberry (Fragaria sp.) grown on colored plastic mulch. Agronomía Colombiana 29(3): 407-413.

10 De Souza L. y Igue T. 1973. Experiência sobre o efeito da cobertura do solo na produção do morangueiro. Bragantia, 32: 149-169.

11 Fernandes-Junior F, Furlani P, Antunes I y Limonta C. 2002. Produção de frutos e estolhos do morangueiro em diferentes sistemas de cultivo em ambiente protegido. Bragantia, 61: 25-34.

12 Gallo E y Santacruz L. 1996. Estudio de adaptabilidad de cinco variedades de fresa, Fragaria sp., industrial de la zona neotropical bajo las condiciones de la Sabana de Bogota. Tesis, Programa de Ingeniería Agronómica, Facultad de Agronomía. Universidad Nacional de Colombia. Bogotá D. C., 137p.

13 García A y Pinilla J. 1998. Evaluación de una cobertura plástica negra en dos variedades de papa (Diacol Capiro y Diacol Monserrate) con dos profundidades de siembra en las localidades de Funza y Suesca. Tesis. Programa de Ingeniería Agronómica, Facultad de Agronomía. Universidad Nacional de Colombia. Bogotá D. C., $72 \mathrm{p}$.

14 González E, Pedraza A y Pérez MM. 2009. Caracterización agrológica del suelo y diagnóstico de su fertilidad en la Estación Experimental Hacienda Río Grande, Cajicá (Cundinamarca, Colombia). Revista Facultad de Ciencias Básicas, 4:82-104.

15 Ham JM, Kluitenberg GJ, Lamont WJ. 1993. Optical properties of plastic mulches affect the temperature regime. Journal of the American Society for Horticultural Science, 118: 188-193.

16 Ibarra L. y Rodríguez A. 1991. Acolchado de suelos con películas plásticas. Editorial Limusa, México, p. 19. 
17 ICONTEC. 1996. Norma Técnica Colombiana NTC 4103, Frutas Frescas, Fresa Variedad Chandler. Editada por el Instituto Colombiano de Normas Técnicas y Certificación ICONTEC, Bogotá, $14 \mathrm{p}$.

18 IDRE-UCLA. 2013. Nonlinear Regression in SAS. http://www.ats.ucla.edu/stat/sas/library/SASNLin_ os.htm, consulta Febrero 2013.

19 Kwabiah AB. 2004. Growth and yield of sweet corn (Zea mays L.) cultivars in response to planting date and plastic mulch in a short-season enviroment. Scientia Horticulturae, 102: 147-166.

20 Laugale V, Bite A y Morocko I. 2006. The Effect of Different Organic Mulches on Strawberries. V International Strawberry Symposium Strawberry Symposium, Acta Horticulturae, 708: 591-594.

21 Montenegro H y Malagón D. 1990. Propiedades físicas de los suelos. Instituto Geográfico Agustin Codazzi, Bogotá, $813 \mathrm{p}$

22 Naranjo P, Padilla M y Pérez MM. 2007. Métodos para estimar el área foliar en plantas de fresa (Fragaria $x$ ananassa) y mora (Rubus glaucus). Memorias 2do. Congreso Colombiano de Horticultura, Sociedad Colombiana de Ciencias Hortícolas, Bogotá, $119 p$.

23 Niu J, Gan Y y Huang G. 2004. Dynamics of Root Growth in Spring Wheat Mulched with Plastic Film. Crop Science, 44: 1682-1688.

24 Poling B. 2005. Silver/black mulch aids strawberries. News of Agribusiness En: Southwest Farm Press, 32: 28. www.southwestfarmpress.com. Consulta Febrero 2008.

25 Pritts M. 2002. Growing strawberries, healthy communities, strong economies and clean environments: what is the role of the researcher? Acta Horticulturae, 567: 411-417.

26 Ramakrishna A, Tam H, Wani S y Long T. 2006. Effect of mulch on soil temperature, moisture, weed infestation and yield of groundnut in northern Vietnam. Field Crops Research, 95: 115-125.

27 Robledo F y Vicente L. 1988. Aplicación de los plásticos en la agricultura. Segunda edición. Mundi-prensa, Madrid. 172 p.

28 Rodríguez G. 2007. Effect of rice bran mulching on growth and yield of cherry tomatoe. Ciencia e investigación agraria, 34: 181-186.

29 SánchezH.2006 Fresa: Requerimientosynormas de cultivo. Proplantas. Mimeografiado. Bogotá. 13 p.

30 Seber G y Wild C. 2003. Nonlinear regression. John Wiley \& Sons, Inc, New Jersey. 768 p.

31 Singh R, Sharma R y Goyal R. 2007. Interactive effects of planting time and mulching on 'Chandler' strawberry (Fragaria $x$ ananassa Duch). Scientia Horticulturae, 111: 344-351.

32 Suárez F y Tovar A. 1997. Efecto de tres tipos de acolchados plásticos en el crecimiento de coles de bruselas (Brassica oleraceae I. var gemmifera) en la sabana de Bogotá y su evaluación económica. Tesis. Programa de Ingeniería Agronómica, Facultad de Agronomía. Universidad Nacional de Colombia. Bogotá D. C.

33 Taiz L y Zeiger E. 2006. Plant Physiology. 4th Edition. Sinauer Associates, Inc., Publishers. Sunderland, Massachusetts. 763 p.

34 Tafur R. 2006. Propuesta frutícola para Colombia y su impacto en la actividad económica nacional, regional y departamental. En: Memorias I Congreso Colombiano de Horticultura. SCCH. Bogotá, Colombia. 1: 47-66.

35 Tenjo H. 2003. Evaluación de diferentes tipos de acolchados plásticos en el desarrollo y productividad de Gypsophila (Gypsophila paniculata L. CV perfecta) cultivada bajo condiciones de invernadero. Tesis. Programa de Ingeniería Agronómica, Facultad de Agronomía. Universidad Nacional de Colombia. Bogotá D. C., 99 p. 